\title{
A Congenital Cause of Recurrent Pneumonia in an Adult
}

\author{
Lana Wahid $^{1 *}$, Bradley Icard ${ }^{2}$, Tomer Pelleg ${ }^{2}$, Susanti Ie $^{2}$ and Anthony Loschner ${ }^{2}$
}

${ }^{1}$ Department of Internal Medicine, Virginia Tech Carilion School of Medicine, USA

${ }^{2}$ Department of Pulmonary, Critical Care and Sleep Medicine, Virginia Tech Carilion School of Medicine, USA

"Corresponding author: Lana Wahid, Internal Medicine Residency, Carilion Clinic-Virginia Tech, Carilion School of Medicine, USA, Tel: 5409817000; Email: 1wahid@carilionclinic.org

\begin{abstract}
Pulmonary sequestration is a rare congenital abnormality especially in the adult population. It can commonly present as a cause for recurrent pneumonia or be found incidentally as an adult on imaging. We present a case of left lower lobe celiac trunk pulmonary sequestration in the setting of recurrent pneumonia to include histoplasmosis.
\end{abstract}

\section{Keywords: Pneumonia; Congenital abnormality; Chest radiography}

Received Date: June 11, 2018; Accepted Date: July 14, 2018; Published Date: July 21, 2018

\section{Background}

Pulmonary sequestration is a rare congenital abnormality especially in the adult population. It can commonly present as a cause for recurrent pneumonia or be found incidentally as an adult on imaging. We present a case of left lower lobe celiac trunk pulmonary sequestration in the setting of recurrent pneumonia to include histoplasmosis.

\section{Case Report}

A 65-year-old man presented with complaints of flu-like symptoms as well as intermittent nausea, vomiting, and diarrhea for two weeks prior, who had been admitted for sepsis secondary to healthcare associated pneumonia. He is a former smoker with a past history significant for histoplasmosis, recurring episodes of pneumonia, and chronic shortness of breath. Prior pulmonary function tests revealed a preserved forced expiratory volume in one second over forced vital capacity ratio with no evidence of restriction despite a mildly reduced diffusion capacity of the lungs for carbon monoxide. Plain film chest radiography demonstrated a large left sided consolidation with an associated pleural effusion. Chest computed tomography with arterial phase angiography demonstrated a soft tissue density in the left lower posterior hemithorax without communicating airways from the tracheobronchial tree [1,2]. The arterial supply originated from the celiac trunk (Figure 1 A-1D). No venous drainage was identified. Thus, the soft tissue density is most consistent with an intralobar pulmonary sequestration. The pleural effusion was sampled which revealed an exudative parapneumonic process and cytology was negative for malignancy. The pleural spaced was drained by tube thoracostomy and the patient was placed on antibiotics [3]. Further laboratory work up included Citation: Lana Wahid, A Congenital Cause of Recurrent Pneumonia in an Adult. J Clin Cases Rep 1(3): 104-106. DOI: https://doi.org/10.46619/joccr.2018.1-1020 
cultures, Legionella antigen, Streptococcus pneumoniae antigen which were all negative. He was discharged home with close follow up.

\section{Discussion}

Pulmonary sequestration is a condition where non-functioning lung tissue is separated from the remainder of the lung and its blood flow is supplied by systemic circulation. This disorder is thought to result from abnormal budding of the primitive foregut and represents approximately 6 percent of all congenital abnormalities of the lower respiratory tract $[4,5]$. There are two forms of pulmonary sequestration, extralobular and intralobular. Extralobular sequestration (ELS) is easily visualized on imaging and tends to be identified early in infancy and can occur along with other congenital abnormalities such as fetal hydrops or polyhydramnios. In ELS, an accessory lung is contained within its own pleura and rarely gets infected. This typically presents in the first six months of life with signs and symptoms of feeding difficulties, chronic cough, abdominal pain, heart failure or respiratory distress. On the other hand, intralobular sequestrations (IPS) traditionally have delayed presentations following recurrent pulmonary infections, hemothorax, pulmonary infarction or hemoptysis. This isolated pulmonary parenchyma is more prone to infection because the lung tissue is contained in the same pleura as the lobe it is developed within but does not have its own bronchial or airway drainage. IPS is more common, representing $75 \%$ of all cases of pulmonary sequestration [6]. There is no association with other congenital anomalies. Although rare, both ELS and IPS can occur simultaneously. Symptomatic pulmonary sequestration can present with complications such as acute respiratory distress syndrome, recurrent pulmonary infections, hypoxemia due to shunting of blood, and even fatal hemorrhage; however, identification and treatment is typically curative with limited morbidity. Managing sequestration can range from observing the asymptomatic patient to surgical resection to prevent complications. Definitive treatment in symptomatic patients involves resection of the affected lung segment.

\section{Conclusion}

We present a rare case of symptomatic left lower lobe celiac trunk intralobar pulmonary sequestration that was diagnosed with an arterial phase chest computed tomography. It is important for clinicians to stay cognizant that intralobar pulmonary sequestration remains in the differential diagnosis of recurrent and non-resolving pneumonia.

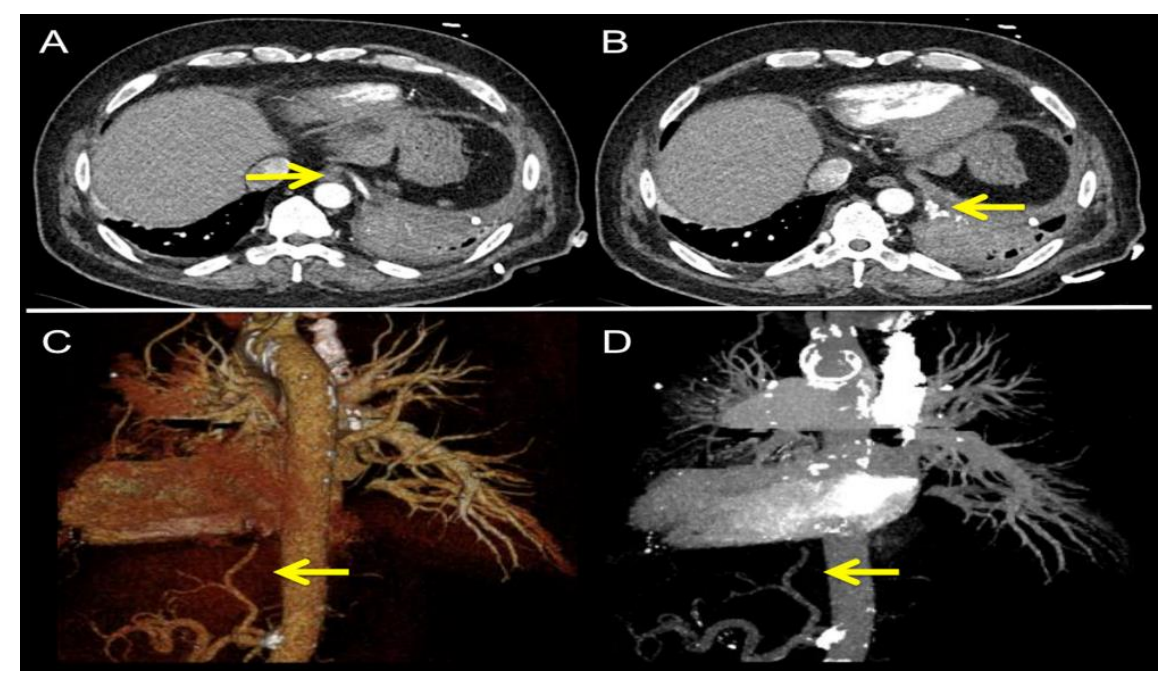

Figure 1: The arterial supply originated from the celiac trunk. 


\section{References}

1. Azizkhan RG and Crombleholme TM (2008) Congenital cystic lung disease: contemporary antenatal and postnatal management. Pediatric Surgery International 24(6): 643-657.

2. Kravitz RM (1994) Congenital malformations of the lung. Pediatric Clinics of North America 41(3): 453-472.

3. https://www.uptodate.com/contents/bronchopulmonary-sequestration

4. Samuel M and Burge DM (1999) Management of antenatally diagnosed pulmonary sequestration associated with congenital cystic adenomatoid malformation. Thorax 54(8): 701-706.

5. Van Raemdonck D, De Boeck K, Devlieger H, et al. (2001) Pulmonary sequestration: a comparison between pediatric and adult patients. European Journal of Cardio-Thoracic Surgery 19(4): 388-395.

6. Akhavan-Heidari M, Edwards D, Besenhaver J et al. (2006) Incidental finding of congenital thoracic malformations in adult population. Southern Medical Journal 99(5): 539-543. 